\title{
Collagen peptides modulate the metabolism of extracellular matrix by human dermal fibroblasts derived from sun-protected and sun-exposed body sites
}

\author{
Vivian Zague, Jonatas Bussador do Amaral, Paula Rezende-Teixeira, Evandro Luis de Oliveira Niero, \\ Camila Lauand and Glaucia Maria Machado-Santelli* \\ Department of Cell and Developmental Biology, Institute of Biomedical Sciences, University of Sao Paulo, Av. Prof. Lineu Prestes, 1524, Sao Paulo, SP \\ 05508-000, Brazil
}

\begin{abstract}
Clinical data published in recent years have demonstrated positive effects of collagen hydrolysate $(\mathrm{CH})$ on skin aging clinical signs. $\mathrm{CH}$ use as food supplement has a long history; however, few studies have addressed the underlying purpose of $\mathrm{CH}$ on the cellular and molecular biology of skin cells that could elucidate clinical improvement findings. Wide diversity of characteristics has been reported for dermal fibroblasts derived from different body sites and it is unknown whether collagen peptides could modulate differently cells from chronological aged and photoaged skin areas. This study investigated the influence of $\mathrm{CH}$ on the extracellular matrix metabolism and proliferation of human dermal fibroblasts (HDFs) derived from chronological aged (sun-protected) and photoaged (sun-exposed) body sites. $\mathrm{CH}$ treatment did not affect cellular proliferation of either cell cultures, but notably modulated cell metabolism in monolayer model, increasing the content of dermal matrix precursor and main protein, procollagen I and collagen I, respectively. These effects were confirmed in the human dermal equivalent model. The increase in collagen content in the cultures was attributed to stimulation of biosynthesis and decreased collagen I metabolism through inhibition of metalloproteinase activity (MMP) 1 and 2. Modulation of CH in dermal metabolism did not differ between cells derived from sun-protected and sun-exposed areas, although lower concentrations of $\mathrm{CH}$ seemed to be enough to stimulate sun-exposed-derived HDFs, suggesting more pronounced effect in these cells. This study contributes to understanding the biological effects of $\mathrm{CH}$ on skin cells and viability of its use as a functional ingredient in food supplements.
\end{abstract}

Keywords: collagen hydrolysate; dermal equivalent; ECM synthesis; MMP-1 activity; photoaged; skin aging

\section{Introduction}

Aging of human skin results from both the passage of time (intrinsic aging) and from cumulative exposure to environmental influences (extrinsic aging). Extrinsic aging affects mostly sun-exposed body areas, while characteristic changes of intrinsically aged skin are mainly visible in sun-protected areas (Fisher et al., 1997; Fligiel et al., 2003; Naylor et al., 2011).

Major structural and functional changes in both the intrinsic and extrinsic aging processes occur in the dermal extracellular matrix (ECM). Dermal ECM is composed of fibrous proteins like collagen, elastin, and fibrillin; proteoglycans like decorin; adhesion molecules and different types of matrix metalloproteinase (MMPs) and play important roles in cell signaling and cellular activities (Tracy et al., 2016). Collagens, specifically type I collagen, are the major components in ECM. Among the numerous modifications of the dermal matrix during aging, alterations in collagen have been suggested as an important cause of clinical changes observed in photoaged and naturally aged skin (Fisher et al., 1997; Fligiel et al., 2003; Naylor et al., 2011).

*Corresponding author: glaucia.santelli@gmail.com

Present address of Jonatas Bussador do Amaral is ENT Research Lab, Department of Otorhinolaryngology—Head and Neck Surgery, Federal University of Sao Paulo, Rua Coronel Lisboa, 958, CEP 04023-900 Sao Paulo, SP, Brazil.

Abbreviations: DE, dermal equivalent; ECM, extracellular matrix; $\mathrm{CH}$, collagen hydrolysate; HDFs, human dermal fibroblasts; MMPs, matrix metalloproteinase 
The influence of dietary supplements on the skin physiology has received increased attention and several clinical studies have indicated that dietary compounds can modulate skin functions (Asserin et al., 2015; Schunck et al., 2015). In parallel to that, in vitro and animal experiments have revealed the action mechanism of food ingredients and their effects on cellular and molecular biology of skin cells. Particularly, collagen hydrolysate $(\mathrm{CH})$ has demonstrated notable stimulatory effect on connective tissue metabolism in cell culture of bovine chondrocytes (Oesser and Seifert 2003). Chen et al. (2017) also showed that these CH can improve intestinal epithelial barrier dysfunction due to its action on tight junctions in vitro model. $\mathrm{CH}$ is a safe and bioavailable dietary supplement composed of low molecular weight $(<6 \mathrm{kDa})$ collagen peptides derived from native collagen by heat denaturation and further enzymatic hydrolysis (Zague 2008; Liu et al., 2015) has been widely used in food supplements and pharmaceuticals. Specific and complex multi-step hydrolysis gives rise to biologically active collagen peptides which have been proven to stimulate skin metabolism and have positive effects on skin properties. Zague et al. (2011) previously reported their beneficial effects on rat skin metabolism by increasing types I and IV collagen expression and reducing MMP-2 activity.

On the basis of clinical studies, daily oral supplementation of specific $\mathrm{CH}$ has been demonstrated to improve skin moisture and elasticity (Proksch et al., 2014b; Inoue et al., 2016) and reduce wrinkle volume in women skin (Proksch et al., 2014a).

A wide diversity of characteristics has been reported for dermal fibroblasts derived from different body sites (Chang et al., 2002), which should be considered when screening for pharmacological or food active ingredients that could modulate cellular properties. The possibility that peptides can cross the intestinal barrier at physically relevant concentrations (Miner-Williams et al., 2015) allows their use in cell culture models.

For this reason, we investigated whether $\mathrm{CH}$ supplementation could affect in a different way cell proliferation and ECM synthesis of skin fibroblasts derived from sunprotected and sun-exposed body sites. In addition, we generated solely human dermal fibroblasts (HDFs)-based dermal equivalent (DE) in order to assess the $\mathrm{CH}$ effects on in vitro three-dimensional model.

\section{Material and methods}

\section{Collagen hydrolysate $(\mathrm{CH})$}

$\mathrm{CH}$ used in this study was supplied by Gelita do Brasil Ltda (Cotia, Brazil), commercially available as Bioactive Collagen Peptides ${ }^{\circledR}$. It was a specific food grade $\mathrm{CH}$, composed of collagen peptides obtained from a complex multistep procedure by degradation and enzymatic hydrolysis of bovine type I collagen. The product was a combination of collagen peptides with mean molecular weight of about $3 \mathrm{kDa}$, previously characterized by Zague et al. (2011).

\section{Cell culture}

Human dermal fibroblasts (HDFs) were derived from skin explants from sun-protected (breast skin) and sun-exposed (eyelid skin) body sites of non-smoking Caucasian women ranging 35-45 years old $(n=6)$. Following informed consent, skin tissue samples were obtained from patients undergoing elective breast and eyelid surgeries. Written informed consent was obtained from each participant and the study was approved by the Clinical Research Ethical Committee of Institute of Biomedical Sciences, University of Sao Paulo; in accordance with current GCP practice regulations and in accordance with the revised Declaration of Helsinki.

The cells were routinely cultured in Dulbecco's modified Eagle's medium (DMEM) with 10\% fetal bovine serum (FBS) (Gibco), penicillin A (100 IU/mL) and streptomycin $(100 \mu \mathrm{g} /$ $\mathrm{mL}$ ). Culture flasks were maintained at $37^{\circ} \mathrm{C}$ in a humidified atmosphere of $5 \% \mathrm{CO}_{2}$. Successive population doublings were obtained with a one to two split ratio. The cells were used between passages three and five. For cell seeding, the vital fibroblasts were counted by means of the trypan blue exclusion method. For the stimulatory assays, cell viability-under $\mathrm{CH}$ treatment-was assessed by MTT viability assay.

\section{Collagen hydrolysate treatment (stimulatory assays)}

For the stimulatory assays, culture medium (10\% FBSDMEM) was replaced by supplemented 10\% FBS-DMEM with $\mathrm{CH}$ at $0.5,1.0,2.5$, and $5.0 \mathrm{mg} / \mathrm{mL}$ (previously determined by MTT viability assay). The control group was kept in $\mathrm{CH}$-free medium. Cell cultures were treated with $\mathrm{CH}$ for $48 \mathrm{~h}$ (previously established).

\section{Cellular proliferation by guava CellGrowth assay (cell division cycle)}

Cellular proliferation was assessed by the Guava CellGrowth Assay (Millipore, Bedford, MA) following the manufacturer's protocol. Acquired data were analyzed in gating dot plot by CytoSoft ${ }^{\mathrm{TM}}$ and expressed as the percentage of proliferating or resting and live or dead HDFs.

\section{Conditioned medium}

For detecting ECM proteins in conditioned medium, fibroblasts were culture $\left(1 \times 10^{5}\right)$ in flasks of $25 \mathrm{~cm}^{2}$ and after 2 days, the medium was removed and the cells were washed exhaustively with PBS, and then placed in FBS-free 
medium supplemented or not with $\mathrm{CH}$. After $48 \mathrm{~h}$ the medium was collected, added to $20 \mu \mathrm{L} / \mathrm{mL}$ of protease inhibitor cocktail (Sigma) (except in the case in which MMPs was evaluated) and dialyzed and concentrated using centrifugal filter device cut-off $30 \mathrm{~K}$ (Amicon Ultra-4, Millipore). Protein amount was determined by BCA assay (Pierce Inc., Rockford, IL, USA).

\section{Type I procollagen and collagen protein expression by immunoblotting}

For the detection of type-I procollagen and collagen in the conditioned medium the use of antibody that recognized the protein in its native soluble form (non-denatured) was needed in order to avoid the detection of collagen peptides from the product used as supplement in the culture medium. Test was done to assure no positive signal for the testproduct. Just prior to loading samples (30 $\mu \mathrm{g} /$ lane) onto precast polyacrylamide gel (NativePAGE Novex 3-12\% Bis-Tris Gel, Invitrogen), $2.5 \mu \mathrm{L}$ of NativePAGE $5 \%$ G-250 sample additive was added to samples on ice. Then, the native gel electrophoresis was run according to manufacturer's instructions. Proteins were transferred to a PVDF membrane which was soaked in the blocking buffer $(25 \mathrm{mM}$ Tris, $150 \mathrm{mM} \mathrm{NaCl}, 2 \mathrm{mM} \mathrm{KCl}, \mathrm{pH} 7.4,0.1 \%$ Tween-20 containing 5\% skimmed milk) for $1 \mathrm{~h}$. The primary mouse monoclonal antibody against type-I procollagen (QED Bioscience Inc.) and type I collagen (Sigma-Aldrich) were used at a dilution of 1:500 in blocking buffer. Secondary antimouse monoclonal antibodies conjugated with horseradish peroxidase (Amersham Inc., Arlington Heights, IL, USA) were diluted to 1:2000 in blocking buffer. The resulting bands were detected by the horseradish peroxidase reaction with an enhanced chemiluminescence kit (Amersham) and exposed to X-ray film (GE Technology).

\section{MMP-1 protein expression by immunobloting}

After measuring protein concentration in conditioned medium, samples were loaded in sample buffer, containing $0.3 \mathrm{M}$ Tris- $\mathrm{HCl}, 5 \%$ SDS, $50 \%$ glycerol, $100 \mathrm{mM}$ dithiothreitol (DTT) (Pierce). Equivalent protein samples $(30 \mu \mathrm{g})$ were subjected to sodium dodecyl sulfate pre-cast polyacrylamide gel electrophoresis (NuPAGE Novex 4-12\% Bis-Tris Gel). Proteins were transferred to a PVDF membrane for $2 \mathrm{~h}$ at $100 \mathrm{~V}$. The membrane was soaked in the blocking buffer (25 mM Tris, $150 \mathrm{mM} \mathrm{NaCl}, 2 \mathrm{mM} \mathrm{KCl}, \mathrm{pH} 7.4,0.1 \%$ Tween-20 containing 5\% skimmed milk) for $1 \mathrm{~h}$. The primary rabbit monoclonal antibody against MMP-1 (Chemicon) was used at a dilution of 1:500 in blocking buffer. Secondary anti-mouse and anti-rabbit monoclonal antibodies conjugated with horseradish peroxidase (Amersham Inc., Arlington Heights, IL, USA) were diluted to
1:2000 in blocking buffer. The resulting bands were detected by the horseradish peroxidase reaction with an enhanced chemiluminescence kit (Amersham Bioscience) and exposed to X-ray film (GE Technology).

\section{Cell-bound type I collagen content by ELISA}

Type I collagen amount in cultured cell layers was determined by indirect ELISA (Collagen Detection Kit, Chondrex, Inc, Redmond, USA), following the manufacturer's protocol.

\section{Type I procollagen subcellular localization}

Cells were seeded $\left(2 \times 10^{4}\right)$ on coverslips in $35 \mathrm{~mm}$-dishes and treated with $\mathrm{CH}$. After $48 \mathrm{~h}$ of treatment, cells were fixed in formaldehyde $3.7 \%$ for $15 \mathrm{~min}$, treated with Triton X-100 for $15 \mathrm{~min}$ and blocked with $1.0 \%$ bovine serum albumin (BSA in PBS) for $30 \mathrm{~min}$. Subsequently cells were incubated with rat monoclonal anti-type I procollagen (1:50 Millipore, USA) overnight at $4^{\circ} \mathrm{C}$. After that, cells were incubated with FITC-anti-rat antibody (1:200) for $90 \mathrm{~min}$. Actin filaments were stained by TRITC-phalloidin. Images were acquired by using a laser scanning confocal microscope (LSM 510, Carl Zeiss Co., Germany).

\section{MMP-1 protein activity}

MMP-1 activity in conditioned medium was measured by Type I Collagenase Activity kit (Chemicon International), following the manufacturer's protocol.

\section{MMP-2 and -9 activities by zymography}

To determine the activity of secreted MMP-2 and -9 in culture media, samples were resuspended in sodium dodecyl sulfate-polyacrylamide gel electrophoresis sample buffer (without $\beta$-mercaptoethanol) (Pierce). Ten micrograms of each sample were loaded in polyacrylamide gels containing $0.2 \%$ gelatin type A from porcine skin (Sigma). After electrophoresis, the gels were washed in $2.5 \%$ Triton X-100

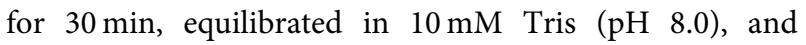
incubated at $37^{\circ} \mathrm{C}$ in a developer buffer containing $50 \mathrm{mM}$ Tris ( $\mathrm{pH} \mathrm{8.0),} 5 \mathrm{mM} \mathrm{CaCl}_{2}$, and $0.02 \% \mathrm{NaN}_{3}$ for $16 \mathrm{~h}$. The gels were stained with $0.2 \%$ Coomassie Brilliant Blue. Gelatinolytic bands were observed as clear zones against the blue background, and the intensity of bands was estimated using Image J software.

\section{Human fibroblast-derived DE and treatment}

Fibroblast-derived matrices (FDMs) were generated according to method proposed by El Ghalbzouri et al. (2009). HDFs 
were seeded into polyester permeable supports at a density of $4 \times 10^{5}$ in each well ( 6 well plates with $0.4 \mathrm{~mm}$ pore size inserts). The FDMs were cultured for 3 weeks in fibroblast medium supplemented with $5 \%$ of fetal bovine serum (FBS) and $100 \mathrm{mg} / \mathrm{mL}$ ascorbic acid phosphate (AAP) under submerged conditions and the culture medium was refreshed every 2 days.

In order to assure that dermal equivalent (DE) was properly formed, culture medium was collected at each refreshment throughout the entire culture period, dialyzed, and concentrated for type I collagen quantification by means of ELISA (as described above). Transmission electron microscopy (TEM) (previously described by Amaral et al. (2010)) was performed at 3 weeks culture to evaluate to what extent the collagen fibers were formed in FDM-based matrix.

For the stimulatory assays (on the 22nd day culture), FDMs were maintained in FBS-free medium supplemented with $\mathrm{CH}$ at 0.5 or $5.0 \mathrm{mg} / \mathrm{mL}$ in association or not with $100 \mathrm{mg} / \mathrm{mL}$ AAP for 24 and $48 \mathrm{~h}$. FDMs in the control group were kept in FBS-free medium supplemented with AAP, but without $\mathrm{CH}$.

\section{Statistical analysis}

Data were analyzed using Minitab software, version 15.1.1.0 (Minitab Inc, State Collage, PA, USA). All variances in the measurement data expressed as mean \pm standard error of mean (SEM) were checked for homogeneity by the Normality's test. Differences among groups were assessed by ANOVA, followed by Tukey-Kramer's multiple comparison post-test. Only $P$-values $<0.05$ were considered statistically significant.

\section{Results}

Effects of $\mathrm{CH}$ on cell-bound type I collagen and ECM proteins expression in conditioned medium

$\mathrm{CH}$ significantly increased $(P<0.05)$ type I collagen amounts in both skin-derived cell cultures, after $48 \mathrm{~h}$ of treatment in contrast to control. The upgrading amount of type I collagen in sun-protected-derived cell cultures was observed after exposure to 2.5 and $5.0 \mathrm{mg} / \mathrm{mL}$ of $\mathrm{CH}$, reaching the highest of 1.3 -fold of control cells. In the sunexposed-derived cells, $\mathrm{CH}$ increased type I collagen amounts in a dose-response manner, being significantly higher than control as of $1.0 \mathrm{mg} / \mathrm{mL}$ and reaching about twofold of control at maximum concentration of $5.0 \mathrm{mg} / \mathrm{mL}$ (Figure 1A).

Representative immunoblottings of ECM proteins expression are shown in Figure 1B. CH treatment of sun-protectedderived cells resulted in notably increased type-I procollagen and collagen expression at doses of 2.5 and $5.0 \mathrm{mg} / \mathrm{mL}$ compared with untreated cells. In sun exposed-derived cells,
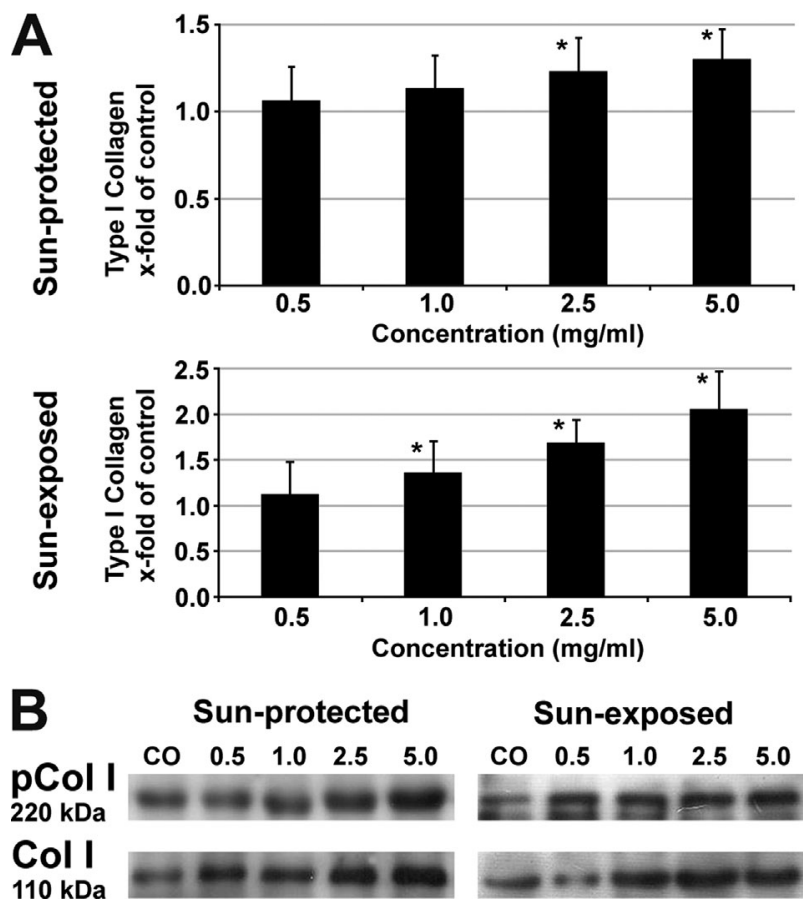

Sun-exposed

Figure 1 Stimulatory effect of collagen hydrolysate $(\mathrm{CH})$ on extracellular matrix (ECM) proteins biosynthesis by human skin fibroblasts (HSFs) derived from sun-protected and sun-exposed body sites. (A) Cell-bound type I collagen amount detected by ELISA after $\mathrm{CH}$ treatment in relation to control. Each column represents the mean \pm SEM value from six independent experiments done in triplicate $\left({ }^{*} P<0.05\right.$, in relation to control group). (B) Representative immunoblotting of type I procollagen ( $\mathrm{pCol} \mathrm{I})$, type I collagen (Col I). Representative immunoblotting from three independent experiments. HDFs were treated with $\mathrm{CH}$ at concentrations ranging from 0.5 up to $5.0 \mathrm{mg} / \mathrm{mL}$ for $48 \mathrm{~h}$ in all the experiments. Control cells (CO) were kept in $\mathrm{CH}$-free medium.

type I procollagen and collagen expression was greatly increased by CH-treatment, as of $1.0 \mathrm{mg} / \mathrm{mL}$, in comparison to control cells.

\section{Type-1 procollagen subcellular localization}

Type I procollagen had the expected perinuclear distribution in control cells from both body sites (Figure 2). Treatment with $2.5 \mathrm{mg} / \mathrm{mL} \mathrm{CH}$ resulted in an increase in both the quantity and distribution of type I procollagen observed in fibroblast derived from both skin sites. This increase was even more evident in cells treated with the highest concentration of $\mathrm{CH}(5.0 \mathrm{mg} / \mathrm{mL})$, showing a noticeable staining bunch around the nuclei, indicating an increase of procollagen biosynthesis promoted by $\mathrm{CH}$ treatment.

\section{Effects of $\mathrm{CH}$ on MMP protein expression and activity}

Type I collagenase (MMP-1) activity in the conditioned medium, from sun-protected and sun-exposed-derived skin 


\section{Sun-protected}
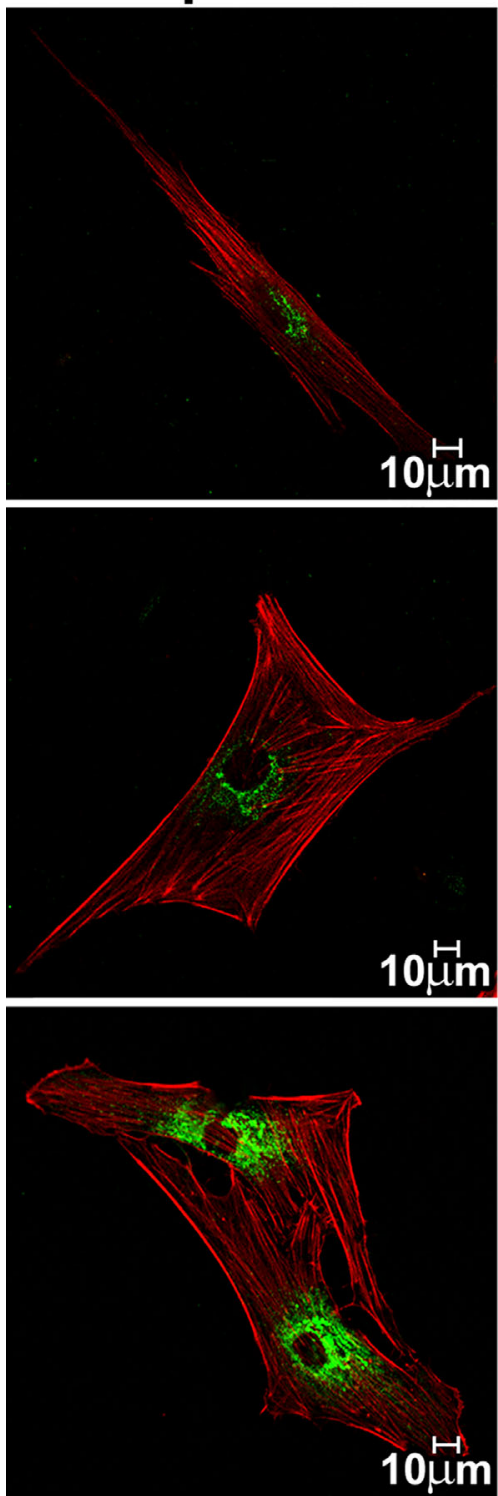

Sun-exposed
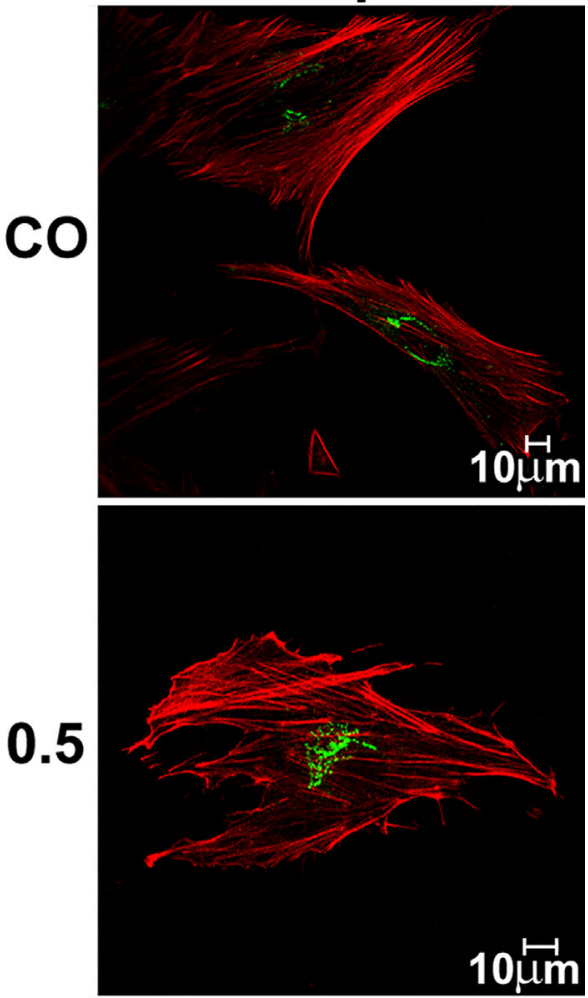

5.0

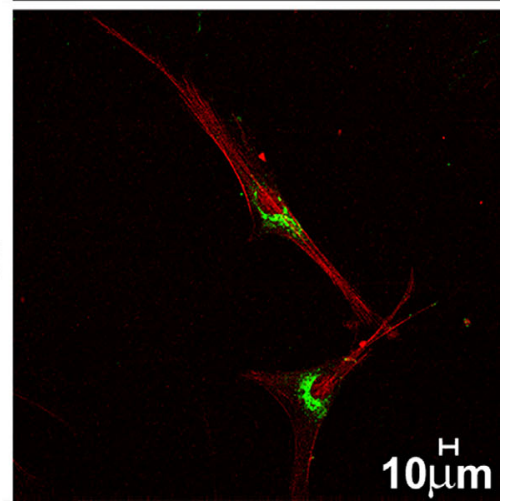

Figure 2 Representative immunostaining of procollagen I subcellular localization in human dermal fibroblasts (HDFs) cultures derived from sun-protected and sun-exposed body sites. HDFs were treated with collagen hydrolysate $(\mathrm{CH})$ at $0.5 \mathrm{and} 5.0 \mathrm{mg} / \mathrm{mL}$ for $48 \mathrm{~h}$. Control cells $(\mathrm{CO})$ were kept in $\mathrm{CH}$-free medium. Positive staining for procollagen I (green) and cytoskeleton (actin filaments) in red.

cells, is demonstrated in Figure 3A. In comparison to untreated cells, $\mathrm{CH}$ treatment led to a dose-dependent decrease in MMP-1 activity by trend. A statistically significant $(P<0.05)$ decrease of collagenase activity could be detected at the highest concentration of $\mathrm{CH}(5.0 \mathrm{mg} / \mathrm{mL})$ compared to the control.

In sun-exposed-derived cells $\mathrm{CH}$ treatment induced a decrease in MMP-1 protein expression at higher concentrations (Figure 3B).

Higher concentrations of $\mathrm{CH}(2.5$ and $5.0 \mathrm{mg} / \mathrm{mL})$ were also associated with a notably decrease in MMP-2 activity
(Figure 3C) compared to untreated cells, in both cell cultures. On the other hand, MMP9 activity was not changed by $\mathrm{CH}$ supplementation in both sun-protected and sunexposed cells.

Solely human fibroblast-derived DE model

A significant and gradual increase in secreted type I collagen was detected during three weeks of HDFs culture in a $3 \mathrm{D}$ model (Figure $4 \mathrm{~A})$. At the end of the third week, sufficient type I collagen amounts $(0.33 \pm 0.07 \mu \mathrm{g} / \mathrm{mL})$ were secreted 
A

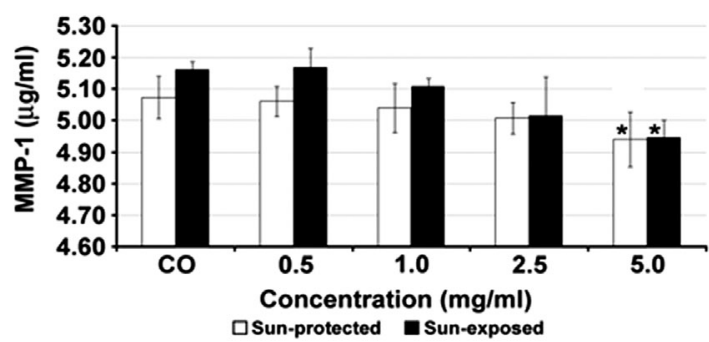

B Sun-protected $\begin{array}{lllll}\text { CO } & 0.5 & 1.0 & 2.5 & 5.0\end{array}$

MMP-1 $72 \mathrm{kDa}$

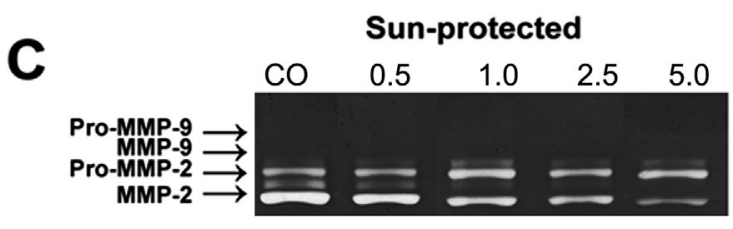

Sun-exposed

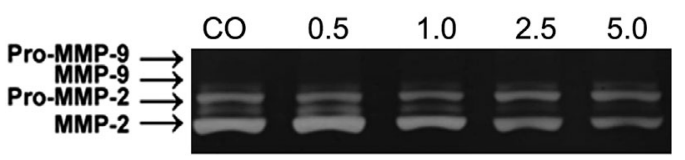

Figure 3 Effects of collagen hydrolysate $(\mathrm{CH})$ on matrix metalloproteinases (MMPs) activity and protein expression by sunprotected and sun-exposed derived human dermal fibroblast (HDFs) cultures. (A) MMP-1 protein amounts in conditioned medium of HDFs cultures treated with $\mathrm{CH}$. Each column represents the mean \pm SEM value from three independent experiments done in duplicate ${ }^{*} P<0.05$, in relation to control group). (B) Representative immunoblotting from three independent experiments of MMP-1 protein expression in conditioned medium. (C) Zymograms for the determination of activities of MMP-2 and -9 in the conditioned medium. Gelatinolytic activities of MMPs 2 and 9 in conditioned medium were detected by electrophoresis of soluble protein on a gelatin-containing $10 \%$ polyacrylamide gel. Representative zymograms were obtained from three independent experiments. HDFs were treated with $\mathrm{CH}$ at concentrations ranging from 0.5 up to $5.0 \mathrm{mg} / \mathrm{mL}$ for $48 \mathrm{~h}$ in all the experiments. Control cells (CO) were kept in $\mathrm{CH}$-free medium.

by fibroblasts to form a functional DE (Figure 4A). The transmission electron microscopy (TEM) revealed a normal assembly of collagen fibrils by the fibroblasts which produced their own matrix, showing the presence of uniform collagen fibrils in the ECM (Figure 4B) and notable network of collagen fibril bundles (Figure 4C).

Stimulatory effects of $\mathrm{CH}$ on human fibroblast-derived DE model

The stimulatory effects of the lowest $(0.5 \mathrm{mg} / \mathrm{mL})$ and highest $(5 \mathrm{mg} / \mathrm{mL})$ concentrations of $\mathrm{CH}$ on the type I collagen secretion in a solely HDFs-DE model can be seen in

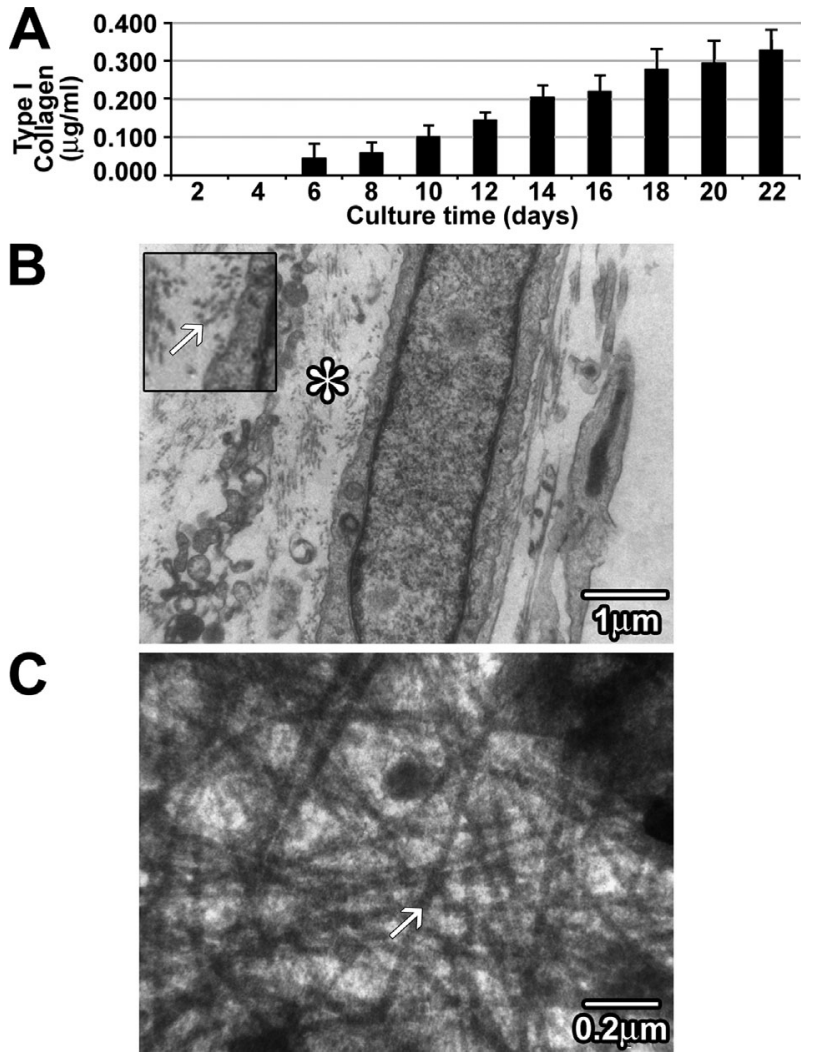

Figure 4 Solely human fibroblast-derived dermal matrices (FDMs). (A) Type I collagen secretion in dialyzed and concentrated culture medium quantified by ELISA. Data demonstrate the amounts of collagen I determined by ELISA at two each day of culture throughout 3 weeks. Bars represent mean \pm SEM of triplicate measurements performed in three independent. (B) Ultrastructure of FDMs assessed by transmission electron microscopy (TEM). Transversal overview of FDMs showing the presence of uniform collagen fibrils marked by the asterisks. (C) General view of FDMs illustrating the notable network of collagen fibrils, indicating their normal processing and assembly. Arrows=collagen fibrils

Figure 5. The lowest concentration of $\mathrm{CH}$ failed to stimulate collagen secretion whilst the highest one significantly $(P<0.05)$ increased collagen secretion compared to control. Cells treated with $5 \mathrm{mg} / \mathrm{mL} \mathrm{CH}$ secreted 1.5 -fold more collagen than the untreated cells. In addition, a pronounced and significant $(P<0.01)$ stimulation of collagen secretion could be observed in cultures treated with $5 \mathrm{mg} / \mathrm{mL}$ associated with ascorbic acid phosphate (AAP), reaching a maximum of 1.8-fold more collagen than control.

\section{Effects of $\mathrm{CH}$ on cellular proliferation}

The treatment with $0.5-5.0 \mathrm{mg} / \mathrm{mL} \mathrm{CH}$ for $48 \mathrm{~h}$ had no significant effect on dermal fibroblast cell division or death, in both skin-derived cell cultures, since the percentage of cells did not differ from control (Table 1). 


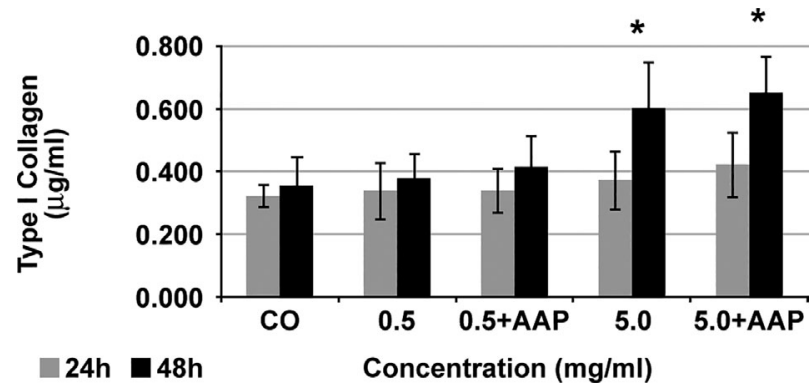

Figure 5 Stimulatory effects of collagen hydrolysate $(\mathrm{CH})$ on type I collagen secretion by solely human fibroblast-derived dermal equivalent model. Type I collagen amounts after 24 and $48 \mathrm{~h}$ of treatment with $\mathrm{CH}$ associated or not with ascorbic acid phosphate (AAP). Control cells (CO) were kept in $\mathrm{CH}$-free medium. Each column represents the mean \pm SEM value from three independent experiments done in triplicate $\left({ }^{*} P<0.05\right.$, in relation to control group).

\section{Discussion}

Fibroblasts are considered the main cell type that synthesizes ECM in connective tissues, nonetheless endogenous and sun-induced aging of the skin cause alterations in cellular morphology, proliferative potential, ECM biosynthesis, and response to growth factors and cytokines, which may appear when skin cells are cultured in vitro (Chung et al., 1996). Consistent with the idea that fibroblasts in vitro have the coordinate regulation and synthesis of ECM characteristic their sites of origin (Chang et al., 2002), to the best of our knowledge, nothing is known as to the potential effects of $\mathrm{CH}$ on the metabolism and proliferation of cultured fibroblasts from different anatomic skin sites.

First, we investigated the influence of $\mathrm{CH}$ on fibroblasts growth and no effect was found on either HDFs derived from sun-protected or sun-exposed sites. $\mathrm{CH}$ did not change the percentage of proliferating fibroblasts in terms of cell division cycle. In parallel, we also carried out proliferating trials assessing cell number counting and number of cells in S-phase trials (data no shown) and $\mathrm{CH}$ also failed to stimulate fibroblast proliferation, allowing us to conclude that $\mathrm{CH}$ could not influence dermal fibroblast growth. Collagen-derived di-peptide (Pro-Hyp) has been reported to induce cell proliferation in human dermal fibroblasts (Ohara et al., 2010a) and cell migration and growth of mouse skin fibroblasts (Shigemura et al., 2009). Apart from the differences between the experimental designs, these authors investigated a single di-peptide normally found in the blood after collagen hydrolysate intake, while we studied the total combination of peptides which $\mathrm{CH}$ is composed of. The biological responses of other collagen-derived peptides (except Pro-Hyp) are also important due to the potential occurrence of high biological activity even at low plasma concentrations, as well as the potential synergistic effects of various peptides. Consequently, skin cells responses can significantly differ when they are exposed to a single collagen-derived peptide or a specific combination thereof (Liu et al., 2015; Inoue et al., 2016), contributing to explain these differences we found.

Besides fibroblast proliferation, we investigated the stimulatory effects of $\mathrm{CH}$ on the secretion of type I collagen, the major protein in dermal ECM. Cell-bound type I collagen was expressively increased by $\mathrm{CH}$ treatment, in sunprotected and sun-exposed-derived cell cultures. $\mathrm{CH}$ treatment led as well to an increase of type I procollagen and collagen amounts in conditioned culture medium, indicating the increase of collagen secretion.

These results are consistent with those reported in animal studies that demonstrate the stimulatory effect of $\mathrm{CH}$ intake on dermal connective tissue metabolism. We previously

Table 1 Effects of collagen hydrolysate $(\mathrm{CH})$ on the percentage of proliferating or resting and live or dead human dermal fibroblasts (HDFs), after 48 h-treatment period. ${ }^{a}$

\begin{tabular}{|c|c|c|c|c|c|}
\hline & \multicolumn{5}{|c|}{$\mathrm{CH}$ concentration $(\mathrm{mg} / \mathrm{mL})$} \\
\hline & $\mathrm{CO}$ & 0.5 & 1.0 & 2.5 & 5.0 \\
\hline \multicolumn{6}{|c|}{$\%$ of cells-sun-protected-derived HDFs } \\
\hline (a) Proliferating & $90.89 \pm 7.85$ & $90.42 \pm 9.76$ & $88.48 \pm 5.44$ & $89.55 \pm 6.78$ & $90.27 \pm 8.96$ \\
\hline (b) Resting & $7.23 \pm 3.40$ & $8.40 \pm 3.69$ & $10.55 \pm 4.91$ & $8.37 \pm 3.75$ & $7.40 \pm 2.25$ \\
\hline (c) Dead, stimulated & $0.23 \pm 0.07$ & $0.62 \pm 0.19$ & $0.43 \pm 0.15$ & $0.38 \pm 0.04$ & $0.17 \pm 0.06$ \\
\hline (d) Dead, resting & $2.66 \pm 1.03$ & $1.55 \pm 0.80$ & $1.53 \pm 0.64$ & $1.73 \pm 0.40$ & $1.17 \pm 0.59$ \\
\hline \multicolumn{6}{|c|}{$\%$ of cells-sun-exposed-derived HDFs } \\
\hline (a) Proliferating & $87.89 \pm 9.58$ & $86.42 \pm 6.30$ & $85.48 \pm 8.41$ & $85.55 \pm 4.76$ & $80.27 \pm 9.36$ \\
\hline (b) Resting & $9.23 \pm 3.45$ & $10.35 \pm 2.69$ & $11.55 \pm 2.50$ & $9.37 \pm 3.41$ & $11.40 \pm 1.15$ \\
\hline (c) Dead, stimulated & $0.23 \pm 0.06$ & $0.42 \pm 0.17$ & $0.43 \pm 0.15$ & $0.38 \pm 0.08$ & $0.18 \pm 0.07$ \\
\hline (d) Dead, resting & $2.64 \pm 0.45$ & $2.32 \pm 0.48$ & $1.53 \pm 0.47$ & $2.20 \pm 0.39$ & $3.17 \pm 0.23$ \\
\hline
\end{tabular}

${ }^{a}$ Percentage \pm SD of (a) CEFS-painted, propidium iodide (PI)-negative, live, proliferating cells; (b) painted, propidium iodide (PI)-negative, live, resting cells; (c) painted, Pl-positive, dead, stimulated cells; and (d) painted, Pl-positive, dead, resting cells. Control cells (CO) were kept in CH-free medium. 
showed that $\mathrm{CH}$ intake stimulated an increase of collagen I and IV amount in rat skin (Zague et al., 2011) and Matsuda et al. (2006) reported an improvement in collagen density in skin of pigs fed $\mathrm{CH}$. Liang et al. (2010) revealed that $\mathrm{CH}$ inhibited collagen loss and collagen fragmentation and increased collagen type I and III protein levels in chronological aged skin of rats.

Interestingly, lower concentrations of $\mathrm{CH}$ were needed to stimulate collagen biosynthesis in sun-exposed cells than sun-protected cells. $\mathrm{CH}$ stimulatory influence was more pronounced in sun-exposed derived fibroblasts. Probable reasons for these findings are based on the metabolism characteristics and ECM biosynthesis that are impaired in sun-exposed-derived cells (Chen et al., 1994; Brinckmann et al., 1995; Krishnan and Birch-Machin, 2006), being more susceptible to external stimuli. Fan et al. (2013) showed that $\mathrm{CH}$ intake could repair the endogenous collagen and elastin protein fibers, and could maintain the natural ratio of type IIII collagen in UV-damaged skin of mice.

This is the first time that stimulatory effects of $\mathrm{CH}$ on type I procollagen expression in human skin fibroblasts has been evaluated by immunofluorescence staining. Our results revealed an improvement in content and distribution of type I subcellular type I procollagen expression promoted by $\mathrm{CH}$ in both fibroblast cultures.

The immunofluorescence images showed the normal perinuclear localization of procollagen in control cells, indicating placement into the endoplasmic reticulum site. At the same time, images of cells treated with higher $\mathrm{CH}$ concentration showed more intense and widespread procollagen staining, suggesting higher transference of procollagen to Golgi apparatus to be secreted. It is well known that type I procollagen is a precursor molecule of mature collagen and its levels generally reflect the levels of collagen biosynthesis (Haukipuro et al., 1991; Oikarinen et al., 1992). Therefore, our immunofluorescence staining findings help to explain the increase of type I procollagen and collagen secretion and deposition in cells cultures.

Then, we investigated whether $\mathrm{CH}$ could affect MMP activity and protein expression and we found a pronounced influence of $\mathrm{CH}$ on decreasing MMP-1 and -2 activities. The decrease in MMP-2 activity by $\mathrm{CH}$ agrees with those we formerly reported in skin of rats (Zague et al., 2011). Liang et al. (2010) also shown that $\mathrm{CH}$ inhibited the age-related increased collagen degradation through attenuating MMP-1 expression and increasing tissue inhibitor of metalloproteinases-1 (TIMP-1) expression in a dose-dependent manner.

It is known that high levels of MMP-1 and -2 not only degrade mature form collagen, but also its precursor newly synthesized and secreted by fibroblasts (Chung et al., 2001). Thus, inhibition of MMP-1 and -2 by $\mathrm{CH}$ decreased the degradation of both collagen and its precursor, increasing the content of these two forms in the culture and promoting procollagen conversion into mature collagen.

Lastly, $\mathrm{CH}$ effects were confirmed in DE model in an attempt to simulate ECM in vivo conditions. The essential fibroblast function of synthesizing ECM was utilized in vitro to produce solely HDFs-based DE, featured by fibroblasts producing their own matrix to form collagen fibril bundles, important structural elements of a fully functional ECM (El Ghalbzouri et al., 2009). The main stimulating additive in these cultures is ascorbic acid phosphate, which promotes the in vitro processing of procollagen to collagen $\alpha$-chains (Traber and Stevens, 2011).

Herein, the highest concentration of $\mathrm{CH}$ significantly induced an increase of collagen secretion and its effects were even more evident in the presence of ascorbic acid phosphate. The outcomes found in the DE model could validate those observed in monolayer model and endorsed us to improve the understanding of the response of dermis tissue to $\mathrm{CH}$ treatment closer to in vivo environment.

These findings also have clinical relevance since they help to explain the existing clinical data found (Proksch et al., 2014a, 2014b). They demonstrated a notable improvement in skin elasticity (Proksch et al., 2014b), significantly decrease in eye wrinkle volume (Proksch et al., 2014a) and markedly increase in collagen I and elastin content (Proksch et al., 2014a) in skin of women who ingested $2.5 \mathrm{~g} /$ day of specific collagen peptides for 8 weeks.

Some mechanisms may be proposed to explain the effects of $\mathrm{CH}$ on ECM metabolism. First, biochemical similarities of $\mathrm{CH}$ to endogenous type I collagen, such as the unique amino acid and peptide profiles, providing amino acids as building blocks as well and bioactive collagen peptides able to modulate protein metabolism (Zague, 2008). Second, collagen peptides from $\mathrm{CH}$ may simulate those presented in damage skin, inhibiting further collagen degradation by MMPs and mitigate indirect inhibition of collagen synthesis by MMP generated collagen degradation. These collagen peptides may function as biological messengers for the underlying cell signaling pathway to help stimulate fibroblasts and trigger the biosynthesis of new collagen fibers and hence the reorganization of the extracellular matrix (Fisher et al., 2009; Liang et al., 2010; Ohara et al., 2010b; Zague et al., 2011). Third, the antioxidative ability of collagen peptides (Mendis et al., 2005a, 2005b) and protective effects on the activities of antioxidant enzymes (Hou et al., 2009; Zhuang et al., 2009) may be an contributory factor to maintain extracellular homeostasis in skin. Finally, it is possible that food-derived collagen peptides may function by binding with the cell surface receptor or transporter, if present, or by affecting the interactions between the extracellular matrix and fibroblasts (Shigemura et al., 2009). 
In view of that (a) fibroblasts derived from skin at different anatomical sites display distinct and characteristic transcriptional patterns which implies in ECM synthesis and (b) the use of oral $\mathrm{CH}$ has been widespread as promising in the prevention and treatment of skin aging signs, this study is a major contribution in understanding the influence of $\mathrm{CH}$ on the metabolism of fibroblasts derived from different skin sites and the viability of its use as a functional ingredient in food supplements.

\section{Acknowledgments and funding}

We are indebted to GELITA do Brasil Ltda, FAPESP, CAPES, and $\mathrm{CNPq}$ for providing all financial and material support for the research. We also thank Dr Ricardo Boggio for his medical support, Roberto Cabado Modia and Marlene de Souza Bernardes for their technical support, Prof. Dr. Alberto de Freitas Ribeiro and Waldir Caldeira for TEM support. This study was supported in part by FAPESP, CAPES, CNPq, and GELITA do Brasil Ltda.

\section{Financial disclosure}

Dr. Zague V is employed by GELITA do Brasil Ltda. The other authors have no conflict of interest.

\section{References}

Amaral JB, Urabayashi SM, Machado-Santelli MG (2010) Cell death and lumen formation in spheroids of MCF-7 cells. Cell Biol Int 34(3): 267-74.

Asserin J, Lati E, Shioya T, Prawitt J (2015) The effect of oral collagen peptide supplementation on skin moisture and the dermal collagen network: evidence from an ex vivo model and randomized, placebo-controlled clinical trials. J Cosmet Dermatol 14(4): 291-301.

Brinckmann J, Açil Y, Wolff HH, Müller PK (1995) Collagen synthesis in (sun-)aged human skin and in fibroblasts derived from sun-exposed and sun-protected body sites. J Photochem Photobiol B 27(1): 33-8.

Chang HY, Chi J-T, Dudoit S, Bondre C, van de Rijn M, Botstein D, Brown PO (2002) Diversity, topographic differentiation, and positional memory in human fibroblasts. Proc Natl Acad Sci USA 99(20): 12877-82.

Chen Q, Chen O, Martins IM, Hou H, Zhao X, Blumberg JB, Li B (2017) Collagen peptides ameliorate intestinal epithelial barrier dysfunction in immunostimulatory Caco-2 cell monolayers via enhancing tight junctions. Food Funct 8(3): 1144-51.

Chen YQ, Bernstein EF, Tamai K, Shepley KJ, Resnik KS, Zhang Hui, Tuan R, Mauviel A, Uitto J (1994) Enhanced elastin and fibrillin gene expression in chronically photodamaged skin. J Invest Dermatol 103(2): 182-6.

Chung JH, Seo JY, Choi HR, Lee MK, Youn CS, Rhie G-e, Cho KH, Kim KH, Park KC, Eun HC (2001) Modulation of skin collagen metabolism in aged and photoaged human skin in vivo. J Invest Dermatol 117(5): 1218-24.

Chung JH, Youn SH, Kwon OS, Eun HC, Kim KH, Park KC, Cho $\mathrm{KH}$, Youn JI (1996) Enhanced proliferation and collagen synthesis of human dermal fibroblasts in chronically photodamaged skin. Photodermatol Photoimmunol Photomed 12(2): 84-9.

El Ghalbzouri A, Commandeur S, Rietveld MH, Mulder AA, Willemze R (2009) Replacement of animal-derived collagen matrix by human fibroblast-derived dermal matrix for human skin equivalent products. Biomaterials 30(1): 71-8.

Fan J, Zhuang Y, Li B (2013) Effects of collagen and collagen hydrolysate from jellyfish umbrella on histological and immunity changes of mice photoaging. Nutrients 5(1): 223-33.

Fisher GJ, Quan T, Purohit T, Shao Y, Cho MK, He T, Varani J, Kang S, Voorhees JJ (2009) Collagen fragmentation promotes oxidative stress and elevates matrix metalloproteinase-1 in fibroblasts in aged human skin. Am J Pathol 174(1): 101-14.

Fisher GJ, Wang Z, Datta SC, Varani J, Kang S, Voorhees JJ (1997) Pathophysiology of premature skin aging induced by ultraviolet light. N Engl J Med 337(20): 1419-29.

Fligiel SEG, Varani J, Datta SC, Kang S, Fisher GJ, Voorhees JJ (2003) Collagen degradation in Aged/Photodamaged skin in vivo and after exposure to matrix metalloproteinase- 1 in vitro. J Invest Dermatol 120(5): 842-8.

Haukipuro K, Melkkocand J, Risteli L, Kairaluoma MI, Risteli J (1991) Synthesis of type I collagen in healing wounds in humans. Ann Surg 213(1): 75-80.

Hou H, Li B, Zhao X, Zhuang Y, Ren G, Yan M, Cai Y, Zhang X, Chen L (2009) The effect of pacific cod (Gadus macrocephalus) skin gelatin polypeptides on UV radiation-induced skin photoaging in ICR mice. Food Chem 115(3): 945-50.

Inoue N, Sugihara F, Wang X (2016) Ingestion of bioactive collagen hydrolysates enhance facial skin moisture and elasticity and reduce facial ageing signs in a randomised double-blind placebo-controlled clinical study. J Sci Food Agric 96(12): 4077-81.

Krishnan KJ, Birch-Machin MA (2006) The incidence of both tandem duplications and the common deletion in mtDNA from three distinct categories of sun-exposed human skin and in prolonged culture of fibroblasts. J Invest Dermatol 126(2): 408-15.

Liang J, Pei X, Zhang Z, Wang N, Wang J, Li Y (2010) The protective effects of long-term oral administration of marine collagen hydrolysate from chum salmon on collagen matrix homeostasis in the chronological aged skin of sprague-dawley male rats. J Food Sci 75(8): H230-8.

Liu D, Nikoo M, Boran G, Zhou P, Regenstein JM, M A-I, D L, MZ F, K S, Y M (2015) Collagen and gelatin transport of a tripeptide, Gly-Pro-Hyp, across the porcine intestinal brushborder membrane. Annu Rev Food Sci 6(1): 527-57.

Matsuda N, Koyama Y-i, Hosaka Y, Ueda H, Watanabe T, Araya $\mathrm{T}$, Irie S, Takehana K (2006) Effects of ingestion of collagen peptide on collagen fibrils and glycosaminoglycans in the dermis. J Nutr Sci Vitaminol 52(3): 211-5. 
Mendis E, Rajapakse N, Byun H-G, Kim S-K (2005a) Investigation of jumbo squid (Dosidicus gigas) skin gelatin peptides for their in vitro antioxidant effects. Life Sci 77(17): 2166-78.

Mendis E, Rajapakse N, Kim S-K (2005b) Antioxidant properties of a radical-scavenging peptide purified from enzymatically prepared fish skin gelatin hydrolysate. J Agric Food Chem 53(3): 581-7.

Miner-Williams WM, Stevens BR, Moughan PJ (2015) Are intact peptides absorbed from the healthy gut in the adult human? Nutr Res Rev 27(2): 308-29.

Naylor EC, Watson REB, Sherratt MJ (2011) Molecular aspects of skin ageing. Maturitas 69(3): 249-56.

Oesser S, Seifert J (2003) Stimulation of type II collagen biosynthesis and secretion in bovine chondrocytes cultured with degraded collagen. Cell Tissue Res 311(3): 393-9.

Ohara H, Ichikawa S, Matsumoto H, Akiyama M, Fujimoto N (2010a) Collagen-derived dipeptide, proline-hydroxyproline, stimulates cell proliferation and hyaluronic acid synthesis in cultured human derma fibroblasts. J Dermatol 37: 330.

Ohara H, Iida H, Ito K, Takeuchi Y, Nomura Y (2010b) Effects of pro-Hyp, a collagen hydrolysate-derived peptide, on hyaluronic acid synthesis using in vitro cultured synovium cells and oral ingestion of collagen hydrolysates in a Guinea pig model of osteoarthritis. Biosci Biotechnol Biochem 74(10): 2096-9.

Oikarinen A, Autio P, Kiistala U, Risteli L, Risteli J (1992) A new method to measure type I and III collagen synthesis in human skin in vivo: demonstration of decreased collagen synthesis after topical glucocorticoid treatment. J Invest Dermatol 98(2): 220-5.

Proksch E, Schunck M, Zague V, Segger D, Degwert J, Oesser S (2014a) Oral intake of specific bioactive collagen peptides reduces skin wrinkles and increases dermal matrix synthesis. Skin Pharmacol Physiol 27(3): 113-9.
Proksch E, Segger D, Degwert J, Schunck M, Zague V, Oesser S (2014b) Oral supplementation of specific collagen peptides has beneficial effects on human skin physiology: a double-blind, placebo-controlled study. Skin Pharmacol Physiol 27: 47-55.

Schunck M, Zague V, Oesser S, Proksch E (2015) Dietary supplementation with specific collagen peptides has a body mass index-dependent beneficial effect on cellulite morphology. J Med Food 18(12): 1340-8.

Shigemura Y, Iwai K, Morimatsu F, Iwamoto T, Mori T, Oda C, Taira T, Park EY, Nakamura Y, Sato K (2009) Effect of prolylhydroxyproline (Pro-Hyp), a food-derived collagen peptide in human blood, on growth of fibroblasts from mouse skin. J Agric Food Chem 57(2): 444-9.

Traber MG, Stevens JF (2011) Vitamins C and E: beneficial effects from a mechanistic perspective. Free Radic Biol Med 51(5): 1000-13.

Tracy LE, Minasian RA, Caterson EJ (2016) Extracellular matrix and dermal fibroblast function in the healing wound. Adv Wound Care 5(3): 119-36.

Zague V (2008) A new view concerning the effects of collagen hydrolysate intake on skin properties. Arch Dermatol Res 300(9): 479-83.

Zague V, de Freitas V, d. C. Rosa M, de Castro Á., Jaeger RG, Machado-Santelli GM (2011) Collagen hydrolysate intake increases skin collagen expression and suppresses matrix metalloproteinase 2 activity. J Med Food 14(6): 618-24.

Zhuang Y, Hou H, Zhao X, Zhang Z, Li B (2009) Effects of collagen and collagen hydrolysate from jellyfish (Rhopilema esculentum) on mice skin photoaging induced by UV irradiation. J Food Sci 74(6): H183-8.

Received 6 July 2017; accepted 3 September 2017. Final version published online 9 October 2017 